\title{
Weichteildefekte an der Hand - die freie mikrovaskuläre Gewebetransplantation
}

\author{
Marc-Daniel Kunze, Isabella Maria Mehling, Michael Sauerbier
}

\section{Zusammenfassung}

Die Behandlung von Weichteildefekten an der Hand stellt einen wesentlichen Teil des handchirurgischen Alltags dar. Die Ätiologie dieser Defekte ist vielfältig und reicht von leichteren traumatischen Ereignissen wie Schnittverletzungen mit Hautverlusten, Ablederungen oder höhergradigen Verbrennungen bis hin zu schweren ausgedehnten Quetschwunden, Hochdruckinjektionsverletzungen, Pfählungen und Amputationsverletzungen. Doch auch akute Infektionen mit nachfolgender Entwicklung chronisch-entzündlicher Wunden sowie komplexe Gewebeverluste nach Tumorresektionen zwingen $\mathrm{zu}$ einer differenzierten Herangehensweise bei der Planung und Ausführung geeigneter plastisch-chirurgischer Operationsverfahren. Hierbei gilt es, wichtige Aspekte hinsichtlich der anatomischen und funktionellen Besonderheiten der Hand im Kontext der individuellen Patientenbedürfnisse zu berücksichtigen.
Das Spektrum an rekonstruktiven Möglichkeiten umfasst dabei sowohl Hauttransplantationen, lokale und gestielte Lappenplastiken als auch technisch höchst anspruchsvolle freie Lappenplastiken mit mikrovaskulärem Anschluss im Empfängergebiet. In diesem Artikel erfolgt eine systematische Einführung in die aktuellen mikrochirurgischen Therapieoptionen bei Weichteildefekten an der Hand. Hierbei werden die wichtigsten Rekonstruktionsschritte ausgewählter freier Lappenplastiken aufgezeigt.

\section{Soft-Tissue Defects of the Hand: Free Microvascular Tissue Transplantation}

The treatment of soft-tissue defects of the hand makes up a considerable part of the daily work at a department specialised in hand surgery. The aetiology of these defects varies and ranges from simple traumatic events, such as cutting injuries with substance defects of the skin envelope, decollements or high-grade burns, to severe and extended squeeze injuries, high-pressure injection injuries, impalements and amputations of different localisations. Furthermore, infections with subsequent development of chronic wounds as well as complex defects after tumour resections require a differentiated approach concerning the planning and the performance of suitable plasticsurgical operative procedures. In the context of individual patients' needs, important aspects concerning the anatomic and functional characteristics of the hand have to be taken into account. The spectrum of reconstructive possibilities encompasses both skin grafting, local and pedicled flaps as well as technically highly demanding free flaps with microvascular connection to the recipient region. This article provides a systematic introduction to the current therapeutic microsurgical options for soft tissue defects of the hand and demonstrates the key steps in the surgical procedure of selected free flaps.

\section{Einleitung}

Defekte im Bereich der Hand und des Unterarms lassen sich auf eine Vielzahl verschiedener Ursachen zurückführen. Patienten mit Unfallverletzungen stellen hierbei einen Großteil des zu behandelnden Patientenguts dar. Die Ausprägungen sind mannigfaltig und reichen von kleineren Defekten im Zuge von Schnittverletzungen bis hin zu massiven Zerstörungen großer Anteile des Weichteilmantels mit Beteiligung wesentlicher Strukturen wie Gefäßen, Nerven, Sehnen und knöchernen Anteilen.

OP-JOURNAL 2015; 31: 124-130

(c) Georg Thieme Verlag KG Stuttgart · New York DOI http://dx.doi.org/10.1055/s-0035-1546078
Häufig sind Defekte im Bereich der Hand jedoch auch auf Infektionen infolge von Weichteilläsionen zurückzuführen, wie sie bspw. durch Phlegmonen nach Tierbissen oder Stichverletzungen durch Pflanzenteile entstehen. Die hierdurch erforderliche operative Entfernung von geschädigten Weichteilen führt nicht selten zum Freiliegen „wertvoller“ Strukturen der Hand wie z.B. Sehnen, Nerven und Gefäßen. Unbehandelt kann es dann zu einem Fortschreiten der Entzündung bzw. einem Austrocknen der betroffenen Strukturen sowie in der Folge einem (teilweisen) Funktionsverlust der Hand kommen. In gleicher Weise spielen benigne oder maligne Tumorerkrankungen, welche sich an der Hand manifestieren, eine wichtige Rolle bei der Indikationsstellung zur Durchführung eines rekon- struktiven Eingriffs nach erfolgter Tumorresektion.

Für die volle Funktionalität der Hand sind Beweglichkeit und Sensibilität von entscheidender Bedeutung. Ein Erhalt dieser Fähigkeiten sollte in Abstimmung mit dem persönlichen Patientenprofil das oberste Ziel der Defektdeckung sein.

Um dieses zu erreichen, haben sich im Laufe der letzten Jahrzehnte signifikante Entwicklungen in den Möglichkeiten der Deckung v.a. mittels freier Lappenplastiken eingestellt. Dabei galt lange Zeit die sog. „Rekonstruktive Leiter“ als das Maß der Dinge, welche eine Auswahl der anzuwendenden Technik zur Defektdeckung im Sinne einer Eskalation von einfachen Verfahren wie dem Primärverschluss über lokale Lappenplastiken bis 
hin zum freien Gewebetransfer vorschlug [1].

Diese Vorgehensweise ist heute jedoch differenzierter zu betrachten, sodass man aktuell eher von in der Indikation gleichwertigen Techniken spricht und die Form der Defektdeckung individuell an die Bedürfnisse des Patienten anpasst [2]. Dieses im Gegensatz zur „Rekonstruktiven Leiter" (reconstructive ladder) als „Rekonstruktiver Fahrstuhl“ (reconstructive elevator) bezeichnete Konzept beschreibt - bildhaft betrachtet das Überspringen komplexer werdender Operationsverfahren zugunsten des sofortigen Einsatzes aufwendiger rekonstruktiver Maßnahmen [3].

Die mikrochirurgische Gewebetransplantation stellt längst nicht mehr die Ultima Ratio in der Behandlung dar, sondern steht als eine von vielen Therapieoptionen dem entsprechend qualifizierten Chirurgen bereits initial zur Verfügung.

Maßgeblich hierfür ist nicht zuletzt die Tatsache, dass freie Lappenplastiken durch grundlegende anatomische Studien und Verbesserung der operativen Techniken wesentlich an Sicherheit in ihrer Anwendung hinzugewonnen haben. Dabei haben klassische Verfahren nach wie vor durchaus ihren Stellenwert in der Behandlung, welche sowohl die konservative Therapie (Vaseline-GazeVerband, Semiokklusivverband etc.) als auch die operative Therapie (Spalthautoder Vollhauttransplantationen, lokale und gestielte Lappenplastiken etc.) umfassen.

\section{Freie Lappenplastiken}

Komplexe Handverletzungen mit größeren Weichteildefekten verlangen oft nach aufwendigen Rekonstruktionsmaßnahmen, welche über die Möglichkeiten lokaler Lappenplastiken hinausgehen. Als limitierend stellt sich dabei oft die Verfügbarkeit von lokalem Gewebe zur Transplantation dar. Zur Rekonstruktion solcher Verletzungen haben sich freie Lappenplastiken bewährt, bei denen Gewebe anderer Körperregionen in das zu deckende Gebiet transplantiert werden. Hierbei wird die Ernährung des transplantierten Gewebes durch einen mikrochirurgischen Anschluss an Gefäße (Arterien und Venen) im Empfängergebiet gewährleistet. Als wesentliche Vorteile freier Lappenplastiken gelten, dass mit ihnen nahezu jede Defektgröße ver- schlossen werden kann, sie über eine eigenständige Blutversorgung verfügen und für einen verbesserten venösen und lymphatischen Abfluss im traumatisierten Bereich sorgen können.

\section{Indikationen}

Als Indikation für den freien Lappentransfer im Bereich der Hand lassen sich im Wesentlichen 2 Gruppen anführen, welche die häufigsten Ursachen für die Wahl dieses Deckungsverfahrens stellen: Dies betrifft in erster Linie die Versorgung nach unfallbedingten Verletzungen, wobei hier die Notfallversorgung innerhalb der ersten Tage nach ausgedehnten Weichteilverletzungen einen wesentlichen Anteil ausmacht [4]. Die andere Gruppe umfasst alle Formen von Tumorerkrankungen am Unterarm und an der Hand. Hier liegt der Fokus v.a. auf malignen Tumoren (z.B. Sarkomen), da die Behandlung primär eine kurative Resektion in sano (R0-Resektion) zum Ziel hat und dementsprechend radikal vorgenommen werden muss.

\section{Notfälle}

Nicht selten entstehen ausgedehnte Defektwunden im Bereich der oberen Extremität trotz verbesserter Sicherheitsvorkehrungen durch berufsbedingte Unfälle bei der Bedienung von Maschinen und Werkzeugen. Doch auch Verletzungen im Rahmen von Freizeitaktivitäten und privaten handwerklichen Tätigkeiten geben immer wieder Anlass zur Indikationsstellung für einen freien Gewebetransfer. Die Rekonstruktion der verletzten funktionellen Strukturen erfolgt hierbei i.d.R. am Unfalltag und schließt insbesondere die Versorgung von Knochenbrüchen, die Adaptation verletzter Sehnen sowie eine Naht oder Rekonstruktion von Gefäßen und Nerven mit ein. Auch die weitere Versorgung solcher schwerwiegender Verletzungen ist anspruchsvoll und sollte daher stets durch ein plastisch-/handchirurgisches Zentrum erfolgen. Hinsichtlich des Zeitpunkts einer Defektdeckung durch mikrochirurgische Rekonstruktion ergeben sich - wie auch für lokale Lappenplastiken - abhängig von defekt- und patientenbedingten Faktoren verschiedene Vorgehensweisen.

Die akute Weichteildefektdeckung mittels freier Lappenplastik (,emergency flap“) nach Polytrauma stellt nach wie vor eine Ausnahmeindikation dar.
Sie ist im Allgemeinen nur bei freiliegenden Gefäßen, glattrandigen Gewebedefekten mit freiliegenden Nerven, bei eröffneten Gelenken und/oder von Knochenstücken (ohne Knochenhaut) oder bei mit intakten Anteilen nicht mehr zu rekonstruierenden Körperabschnitten sinnvoll. Der Vorteil liegt insbesondere in der Vermeidung eines weiteren Gewebeschadens durch Austrocknung oder Superinfektion. Dies muss jedoch mit dem Gesamtzustand des Patienten abgewogen werden, weshalb sich oft eine länger dauernde Operation am Unfalltag verbietet. Die verzögerte akute Defektdeckung im Verlauf von 24-72 Stunden nach Trauma trägt der Tatsache Rechnung, dass die wahre Ausdehnung des vorliegenden Weichteilschadens oft erst nach sichtbarer Abgrenzung geschädigter Areale erkennbar ist. Marko Godina hat dies bereits 1986 mit beeindruckenden Patientenzahlen postuliert [5]. Die Primärversorgung erfolgt daher durch eine zeitlich begrenzte Deckung mittels Kunsthaut oder durch Vakuumverbände nach Säuberung der geschädigten und kontaminierten Weichteile. Dies gilt jedoch nur für den Fall, dass keine Sehnen in der Wunde freiliegen. Hier ist bei der temporären Versorgung der Wunde (z.B. mit grobporigen Schaumstoffverbänden) unbedingt eine kontinuierliche Befeuchtung des Gewebes erforderlich, um ein Austrocknen der Sehnen und ggf. des umgebenden Sehnengleitgewebes $\mathrm{zu}$ vermeiden. Im Zuge von erfolgten Infektoder Weichteilsanierungen als Vorbereitung für weitere Operationen werden im Allgemeinen sekundäre Defektdeckungen vorgenommen.

\section{Tumorerkrankungen}

Patienten mit Tumorerkrankungen, meist Weichgewebesarkomen, weisen im Verlauf der erforderlichen radikalen Therapiemaßnahmen nicht selten erhebliche Weichteildefekte im betroffenen Gebiet auf. Als Standardeingriff hat sich gerade bei Weichgewebesarkomen die weite Exzision („wide excision“) des Tumors etabliert. Dies führt jedoch insbesondere im Bereich der Extremitäten nicht selten zu einem massiven Gewebeverlust, der oft auch die notwendige Amputation von Anteilen der Gliedmaße bedeutet. Anhand von aktuellen Studien konnte für Weichgewebesarkome jedoch gezeigt werden, dass sogar die Entfernung mit einem Sicherheitsabstand von unter $1 \mathrm{~cm}$ zum Tumorrand im Hinblick auf die Vorbeugung eines möglichen Tumorrezidivs ausreicht und damit für den 
Funktionserhalt der betroffenen Extremität eine wesentliche Bedeutung hat [6]. Derart geringe Sicherheitsabstände beinhalten jedoch häufig strategisch, dass der Tumorresektion zumindest bei G2/G3-Tumoren eine Bestrahlung (Radiatio) folgt. Diese Vorgehensweise ist Teil einer bei Sarkomen üblichen multimodalen Therapie, deren fester Bestandteil eine plastisch-chirurgische Rekonstruktion sein sollte [7].

\section{Klassifikation}

Prinzipiell stehen für die Gewebetransplantation aus verschiedenen Spenderregionen des Körpers mehrere Formen freier Lappenplastiken zur Verfügung. Hierbei wird u.a. hinsichtlich der Gewebearten unterschieden, aus welchen das zum Transfer vorgesehene Gewebe aufgebaut ist (s. Tab. 1). Dabei gehen in die Lappenbezeichnung diejenigen Komponenten ein, welche als zusammenhängendes Gewebestück für die Transplantation in das Empfängerareal entnommen werden. Eine freie Haut-Muskel-Lappenplastik (muskulokutane Lappenplastik) bezeichnet demnach die Kombination aller 3 genannten Gewebearten, die gemeinsam von einer versorgenden Arterie und begleitenden $\operatorname{Vene}(\mathrm{n})$ durchblutet werden.

Viele Spenderareale von freien Lappenplastiken gestatten nicht nur eine Variabilität in der Flächenausdehnung des entnommenen Transplantats, sondern auch hinsichtlich der Tiefe und damit der für die Transplantation vorgesehenen Gewebearten.

Gemeinsam ist allen genannten Lappenarten die relativ konstante Versorgung der transplantierten Körperareale durch anatomisch definierte Gefäßäste. Das durch diese mit Blut perfundierte Gebiet ist naturgemäß in seiner Ausdehnung begrenzt und weist für die einzelnen regionalen Lappentypen unterschiedliche maximale Entnahmegrößen auf, welche zudem interindividuellen Schwankungen unterliegen.

Im Unterschied zu den „klassischen“ (Muskel-)Lappenplastiken handelt es sich bei den sog. Perforatorlappen um Lappenplastiken, die aus Haut, Subkutangewebe und - in der Regel - einem Faszienanteil ohne Muskelgewebe bestehen. Der zugrunde liegende Muskel bleibt hierbei charakteristischerweise in situ und wird, möglichst unter Schonung der den Muskel innervierenden Nerven,
Tab. 1 Nomenklatur freier Lappenplastiken.

Haut-Unterhaut-Lappenplastik (adipokutane Lappenplastik)

Haut-Faszien-Lappenplastik (fasziokutane Lappenplastik)

\begin{tabular}{l}
\hline Faszienlappenplastik \\
\hline Muskellappenplastik \\
\hline $\begin{array}{l}\text { Haut-Muskel-Lappenplastik (muskulokuta- } \\
\text { ne Lappenplastik) }\end{array}$ \\
$\begin{array}{l}\text { Haut-Muskel-Knochen-Lappenplastik (os- } \\
\text { teo-[septo]-muskulokutane Lappenplastik) }\end{array}$
\end{tabular}

Perforatorlappenplastik

gänzlich belassen [8]. Diese Präparationsweise erlaubt eine deutliche Reduktion des Hebedefekts mit seinen funktionellen Einschränkungen und bietet gerade bei der Wiederherstellung im Extremitätenbereich hervorragende Einsatzmöglichkeiten entsprechend der hier relevanten funktionellen und ästhetischen Anforderungen.

\section{Sonderformen und funktioneller Ersatz durch freie Lappenplastiken}

Bei vielen freien Lappenplastiken mit Hautanteilen ist der zusätzliche Anschluss eines sensiblen Nervenasts möglich. Hierdurch kann in ausgewählten Fällen zumindest eine Schutzsensibilität im transplantierten Areal erzielt werden, auch wenn die mögliche erreichbare Wiederherstellung der Berührungsempfindlichkeit i.d.R. nicht der gleichen Qualität eines normalen Hautareals entspricht. Die neurovaskuläre Anbindung eines Transplantats bietet jedoch nicht nur die Möglichkeit einer sensiblen Innervierung der wiederhergestellten Areale: Die mikrochirurgische Nervenund Gefäßanbindung eines transplantierten Muskels kann zu einer motorischen Rekonstruktion im Empfängergebiet genutzt werden, um zerstörtes oder tumorbedingt reseziertes Muskelgewebe auch funktionell zu ersetzen (freie funktionelle Muskeltransplantation) [9]. Meistens findet diese Technik jedoch ihre Anwendung zur Behandlung neuromuskulärer Insuffizienzen wie bspw. nach Nervenläsionen im Bereich des Armplexus.

Zusätzliche rekonstruktive Optionen ergeben sich, wenn die zur Transplantation ausgewählte Lappenplastik die Mitnahme eines knöchernen Anteils (z.B. des
Schulterblatts) erlaubt. Ein auf diese Weise in das Empfängergebiet eingebrachtes ossäres Gewebe bietet im Vergleich zu herkömmlichen avaskulären Knochenspänen oder einer Spongiosaplastik eine hervorragende Gewebeperfusion, die durch den mikrovaskulären Anschluss der Lappenhauptgefäße erreicht wird. Auch die alleinige Verpflanzung von vaskularisierten Knochenspänen ist möglich und bietet sich insbesondere bei Defekten nach Unfällen oder nach Resektion entzündlicher Knochenanteile bei Osteomyelitis an, welche $\mathrm{zu}$ diesem Zweck oft erst nach bereits erfolgter (mikrochirurgischer) Defektdeckung bei stabilen Weichteilverhältnissen in einer 2. Operation vorgenommen wird.

\section{Ausgewählte freie Lappenplastiken}

Nachfolgend sollen nun einige der wichtigsten freien Lappenplastiken für die Defektdeckung nach ausgedehnten Verletzungen im Bereich der Hand näher beschrieben werden (s. Tab. 2). Als Operateur gilt es grundsätzlich, den jeweils zu versorgenden Defekt anhand seiner Größe, der freiliegenden Strukturen und funktionellen Anforderungen sowie unter Beachtung der individuellen Anforderungen des Patienten zu beurteilen und entsprechend der gegebenen körperbezogenen und auch technischen Möglichkeiten die adäquate Lappenplastik auszuwählen.

\section{Paraskapular- und Skapulalappen}

Gerade bei schlanken Patienten mit dünner subkutaner Fettschicht sind Paraskapular- und Skapulalappen eine gute Wahl zur Defektdeckung größerer Weichteildefekte im Bereich der Hand [10]. Wie bereits oben erwähnt, ist die Hebung der genannten Lappen auch als osteokutane Variante möglich. Damit bieten sich diese Lappenplastiken ebenso für die Deckung von kombinierten Haut-, Weichteil- und Knochendefekten an. Die Gefäßversorgung erfolgt über das Stromgebiet der A. subscapularis, welche ihren Ursprung in der A. axillaris hat. Im Falle einer Paraskapularlappenplastik erfolgt die Planung der zu entnehmenden Hautspindel längs verlaufend über dem lateralen Rand des Schulterblatts. Der Gefäßverlauf der versorgenden Lappenarterien muss zuvor am sitzenden Patienten durch eine Doppler-Untersuchung dargestellt werden (s. Abb. 1). Die Präparation der Skapulalappenplastik gleicht in ihrer Gefäßdarstellung der Paraskapularlappenplastik, verläuft ent- 
Tab. 2 Beispiele für freie Lappenplastiken zur Defektdeckung an der Hand.

\begin{tabular}{|c|c|c|c|}
\hline Bezeichnung & Art des Lappens & Spenderareal & Stromgebiet \\
\hline $\begin{array}{l}\text { Paraskapular- und } \\
\text { Skapulalappen }\end{array}$ & $\begin{array}{l}\text { kutaner, adipokutaner, fasziokutaner oder } \\
\text { osteokutaner Lappen }\end{array}$ & rückseitige Schulterregion & A. subscapularis \\
\hline lateraler Oberarmlappen & $\begin{array}{l}\text { fasziokutaner, osteofasziokutaner Lappen; } \\
\text { auch als Faszienlappen }\end{array}$ & seitlicher Oberarm & A. radialis collateralis posterior \\
\hline Radialis-Faszienlappen & Faszienlappen & Vorderseite des Unterarms & A. radialis \\
\hline Musculus-gracilis-Lappen & $\begin{array}{l}\text { Muskellappen (auch funktionell), muskulo- } \\
\text { kutaner Lappen (nicht an der Hand) }\end{array}$ & $\begin{array}{l}\text { Innenseite des } \\
\text { Oberschenkels }\end{array}$ & A. circumflexa femoris medialis \\
\hline Leistenlappen & fasziokutaner, osteofasziokutaner Lappen & seitliche Leistenregion & A. circumflexa ilium superficialis \\
\hline $\begin{array}{l}\text { ALT-Lappen (anterolateraler } \\
\text { Oberschenkellappen) }\end{array}$ & $\begin{array}{l}\text { Perforatorlappen (evtl. mit Muskel, } \\
\text { Oberschenkelfaszie) }\end{array}$ & $\begin{array}{l}\text { Außenseite des } \\
\text { Oberschenkels }\end{array}$ & $\begin{array}{l}\text { Perforansgefäße der A. circum- } \\
\text { flexa femoris lateralis }\end{array}$ \\
\hline
\end{tabular}
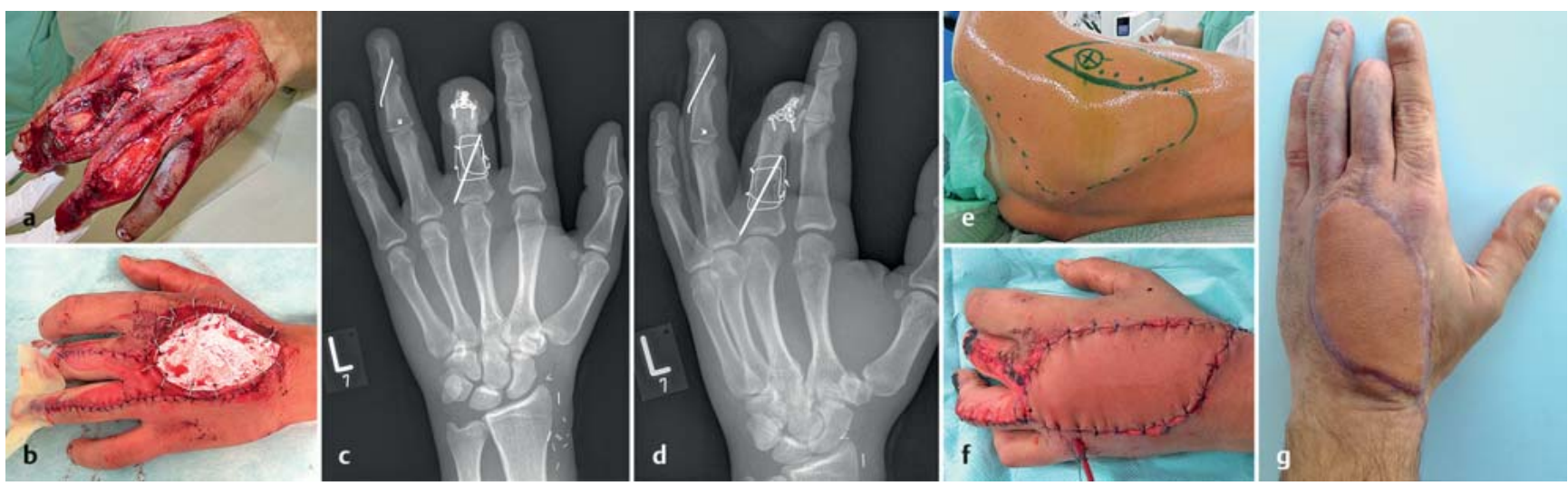

Abb. 1 a bis $\mathbf{g}$ Paraskapularlappen: a Schleifmaschinenverletzung der linken Hand eines 27-jährigen Patienten mit ausgedehnten Weichteil- und Strecksehnenverletzungen sowie multiplen Frakturen. b Befund nach erfolgter Primärversorgung der verletzten Strukturen und temporärer Weichteildeckung durch Kunsthaut. $\mathbf{c}$ und d Radiologischer Befund nach Osteosynthese. e Präoperative Anzeichnung der Lappenentnahmestelle. f Unmittelbar postoperativer Befund nach Lappendeckung am 5. Tag nach Unfall. g Verlaufsbefund 3 Monate postoperativ.

sprechend ihrer versorgenden Gefäße jedoch quer über das Schulterblatt.

Unerwähnt darf in diesem Zusammenhang nicht die Musculus-latissimus-dorsi-Lappenplastik bleiben, welche insbesondere bei ausgedehnten Defekten ihren Einsatz findet. Auch sie stammt aus dem Versorgungsgebiet der A. subscapularis und bietet als freie Haut-Muskel-Lappenplastik, Muskel-Lappenplastik (auch frei funktionell), Haut-MuskelKnochen-Lappenplastik oder sogar als Perforanslappenplastik eine Vielzahl an Anwendungsmöglichkeiten.

\section{Lateraler Oberarmlappen}

Der laterale Oberarmlappen bietet sich für eine Defektdeckung an der Hand in besonderem Maße an, da er dünnes, weiches und gut einpassbares Gewebe für eine Transplantation liefert (s. Abb. 2) [11]. Neben einer hohen anatomischen Konstanz des Gefäßstiels bietet diese Lappenplastik eine relativ geringe Hebe- morbidität, sofern ein Primärverschluss (bei einer Lappenbreite von maximal $6 \mathrm{~cm}$ ) erreicht wird. Als Modifikation ist dieser Lappen zudem als reine Faszienlappenplastik planbar und lässt auch die Mitnahme von Sehnenanteilen des M. triceps oder Anteilen des Oberarmknochens zu (osteofasziokutane Lappenplastik). Die arterielle Versorgung des Spenderareals stammt aus der A. radialis collateralis posterior, welche keine dominante Arterie des Arms darstellt und daher für die Perfusion der oberen Extremität verzichtbar ist. Als Besonderheit bietet der laterale Oberarmlappen die Möglichkeit eines sensiblen Anschlusses des im Lappenbereich verlaufenden $\mathrm{N}$. cutaneus brachii posterior im Empfängergebiet. Bei der Präparation ist unbedingt auf eine Schonung des N. radialis zu achten, der entlang des Oberarmknochens durch das Operationsgebiet zieht.

\section{Freie Faszienlappen}

Gerade im Handbereich, wo es auf gute funktionelle und ästhetisch ansprechende Ergebnisse ankommt, sind Faszienlappen eine elegante Alternative zu den mitunter recht auftragenden Resultaten bei adipösen Patienten. Mit ihrem dünnen und geschmeidigen Gleitgewebe sind sie in besonderem Maße dazu geeignet, exponierte Strukturen wie Sehnen oder Nerven suffizient zu bedecken [12]. Als Beispiele seien hier der freie Musculus-serratus-anteriorFaszienlappen, der freie temporoparietale Faszienlappen und der freie Radialisfaszienlappen erwähnt, welche auch als retrograd gestielte Variante eine ansprechende und sicher durchzuführende Deckung größerer Areale im Handbereich ermöglicht [13]. Gemeinsam ist den Faszienlappenplastiken - wie auch den Muskellappenplastiken - die Erforderlichkeit einer Deckung des transplantierten Gewebes mit Spalthaut, welche aufgrund der hervorragenden Perfusion 


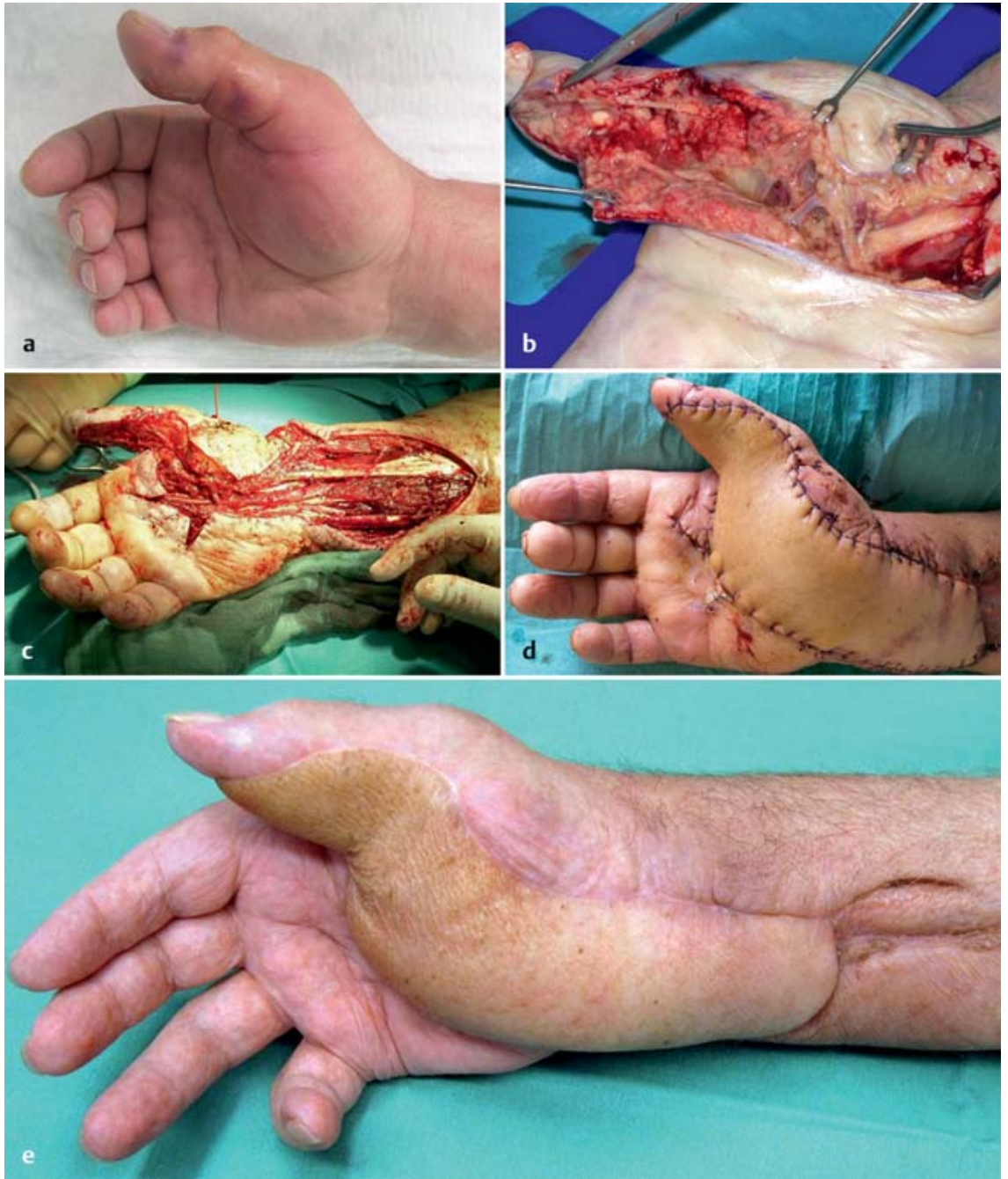

Abb. 2a bis e Freier lateraler Oberarmlappen: a Nekrotisierende Fasziitis im Bereich des rechten Daumens bei einem 70-jährigen Mann. b Intraoperativer Befund am Aufnahmetag. c Ausgedehnter Weichteildefekt nach serieller Wundreinigung. d Postoperativer Befund nach Defektdeckung. e Abschließendes Ergebnis nach kompletter Einheilung des Lappens.

des neu gewonnenen Untergrunds im Allgemeinen gut angenommen wird.

\section{Musculus-gracilis-Lappen}

Diese Lappenplastik gehört aufgrund ihrer konstanten Gefäßversorgung und der geringen Morbidität im Bereich der Entnahmestelle sicherlich zum Standardrepertoire in der Behandlung von Weichteildefekten an der Hand. Gerade auf dem Handrücken hat sich diese Lappenplastik bewährt, da der Muskel in seiner Dicke stark schrumpft und somit kaum aufträgt. Eine Hebung ist prinzipiell auch als myokutaner Lappen möglich, findet im Bereich der oberen Extremität aufgrund der daraus resultierenden Schichtdicke in dieser Form jedoch meist keine Anwendung. Der M. gracilis wird aus der A. circumflexa femoris medialis versorgt. Durch Mitnahme des den Muskel versorgenden Nervenasts kann der Musculus-gracilis-Lappen im Empfängergebiet auch als freie funktionelle Lappenplastik eingesetzt werden.

\section{Leistenlappen}

Der Leistenlappen war einer der ersten Lappen, welcher für die freie mikrochirurgische Gewebetransplantation genutzt wurde [14]. Als gestielte Leistenlappenplastik wurde er bereits in den frühen 1970er-Jahren zur Defektdeckung ausgedehnter Weichteil- und Knochendefekte im Bereich der Hand beschrieben [15] und findet auch heute noch Anwendung, insbesondere in der Versorgung kindlicher Verletzungen. Hier bietet sich der Leistenlappen als sicheres Spenderareal in anatomisch günstiger Position an, wobei gerade bei Kindern das Risiko für eine dauerhafte Bewegungsein- schränkung aufgrund der erforderlichen 3-wöchigen Immobilisierung zugleich als niedrig einzustufen ist. Erst nach diesem Zeitraum ist eine sichere Durchtrennung des Lappenstiels möglich, die durch wiederholtes Abklemmen der versorgenden Weichteilbrücken als sog. „Lappenkonditionierung“ vorbereitet wird. Als freie Lappenplastik hat der Leistenlappen im Laufe der Jahre für die Handrekonstruktion etwas an Bedeutung verloren, da mittlerweile dünnere Lappenplastiken mit längerem (und dickerem) Gefäßstiel sowie geringerem Präparationsaufwand zur Verfügung stehen. Seine Blutversorgung erhält der Leistenlappen aus der A. circumflexa ilium superficialis, welche von der $A$. femoralis ausgehend knapp unterhalb und parallel des Leistenbands nach außen auf die Spina iliaca anterior superior zuläuft. Als Variante lässt sich der Leistenlappen auch mit einem Knochenspan aus dem Beckenkamm als osteofasziokutane Lappenplastik heben, womit sich insbesondere Defekte der Mittelhandknochen rekonstruieren lassen.

\section{Fasziokutaner anteriorer lateraler Oberschenkellappen (,anterolateral thigh flap“, ALT-Lappen)}

Ein gängiges Verfahren für die Weichteildeckung an der Hand stellt heute der sog. ALT-Lappen dar, welcher mehrere Vorteile sowohl hinsichtlich seiner Deckungsqualitäten als auch bez. des Hebedefekts bietet [16]. An der vorderen seitlichen Fläche des Oberschenkels in Projektion auf den M. vastus lateralis gelegen bietet er prinzipiell einen ästhetisch günstigen Narbenverlauf, auch wenn einige Autoren die Indikation bei Frauen eher zurückhaltend stellen. Auch bei adipösen Patienten ist der Lappen insbesondere im Handbereich für eine Deckung als ungünstig zu werten. Der ALT-Lappen ermöglicht eine protektive Sensibilität im Empfängergebiet durch Anschluss des hier verlaufenden N. cutaneus femoris lateralis. Weitere Vorzüge bestehen in der Option der Hebung u.a. als Faszienlappenplastik, funktionelle Muskellappenplastik oder als sog. chimäre Lappenplastik in Verbindung mit weiteren Komponenten des Entnahmegebiets, wie bspw. dem M. rectus femoris oder der anteromedialen Oberschenkelhaut. Seine Blutversorgung erhält der ALT-Lappen als Vertreter der Perforanslappenplastiken in der überwiegenden Mehrheit von muskulokutanen und in geringerer Anzahl auch septokutanen Perforansgefäßen des R. descendens der 


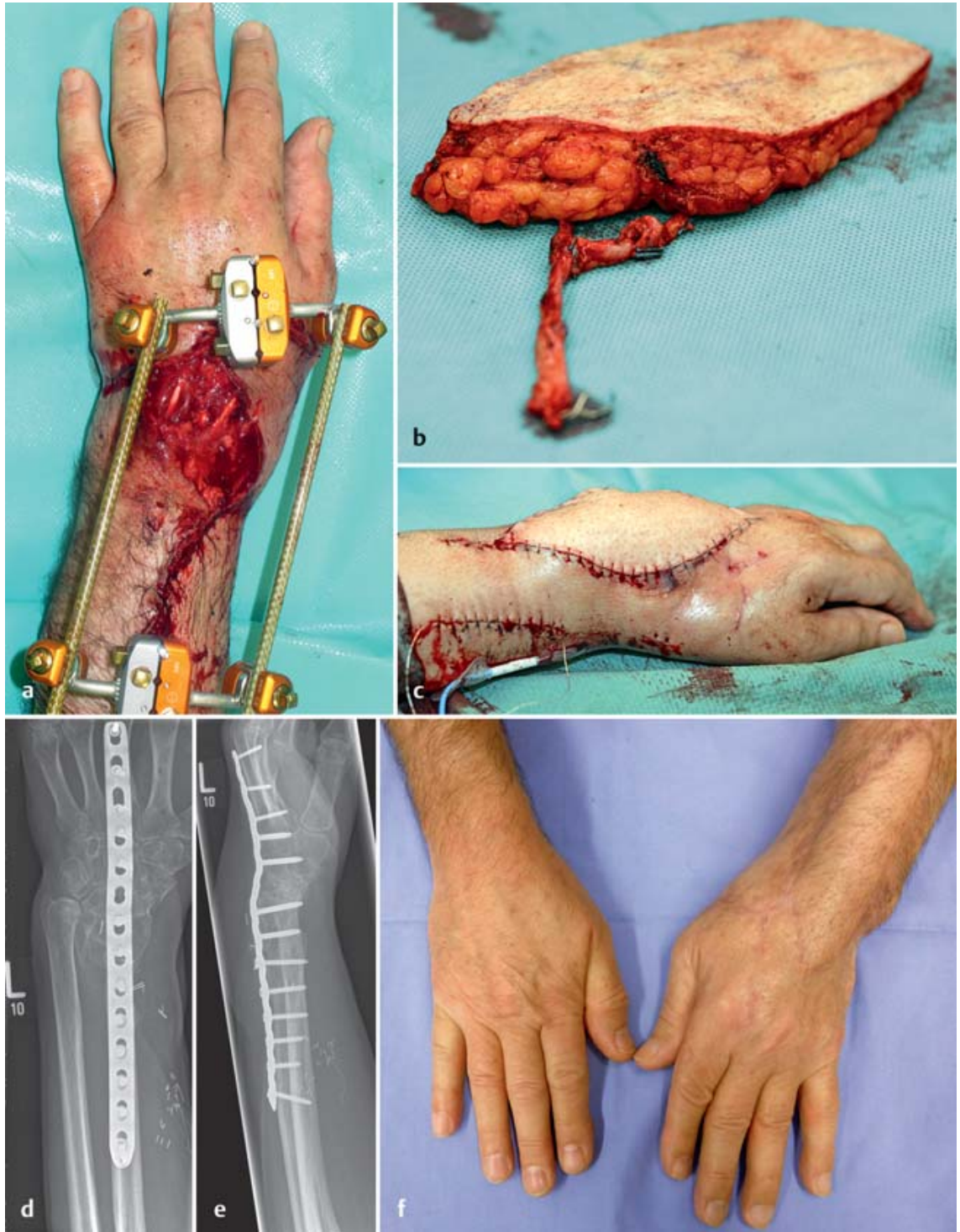

Abb. 3a bis $\mathbf{f}$ ALT-Lappenplastik: a Ausgedehnte Quetschverletzung und Weichteildefekt der linken Hand eines 62-jährigen Patienten mit offenen Luxationsfrakturen des distalen Radius und der Handwurzel. b Vom rechten Oberschenkel entnommener ALT-Lappen mit den versorgenden Lappenstielgefäßen. c Unmittelbar postoperativer Befund nach Defektdeckung. d, e Radiologischer Befund bei erfolgter Handgelenksversteifung und Abnahme des Fixateur externe. $f$ Verlaufsbefund 8 Monate postoperativ.

A. circumflexa femoris lateralis. Im Rahmen der präoperativen Vorbereitung gilt es, mithilfe einer Doppler-Sonografie die Perforansgefäße aufzusuchen und zu markieren (s. Abb. 3).

\section{Schlussfolgerung}

Die plastische Deckung ausgedehnter Weichteildefekte im Bereich der Hand stellt nach wie vor eine große Herausforderung für den behandelnden Chirurgen dar. In diesem Zusammenhang haben sich in zunehmendem Maße freie Lappenplastiken etabliert, welche ihren berechtigten Stellenwert mittlerweile auch in der frühen rekonstruktiven Phase nach Unfällen oder in der Wiederherstellung infolge von Tumoroperationen haben. Hierbei gilt es, entsprechend der speziellen Anforderungen im Handbereich hinsichtlich Funktion und Ästhetik die für den Patienten individuell angemessene Form der mikrochirurgischen Defektdeckung auszuwählen. Unterschiede bei den zur Verfügung stehenden freien Lappenplastiken liegen neben dem resultierenden Hebedefekt u. a. in der maximal erreichbaren Größe und der Gewebeart (Haut, Unterhaut, Muskel und Knochen) des Spenderareals. Als Ergänzungsmöglichkeit bie- ten manche Lappenarten den sensiblen Anschluss im Empfängergebiet oder den funktionellen Ersatz von Muskelgewebe.

Sowohl der aufwendige operative Eingriff mit mikrochirurgischer Verbindung von Gefäßen als auch die postoperative Nachbehandlung erfordern besondere Voraussetzungen und Kenntnisse, welche die Behandlung der Patienten in einer hierfür spezialisierten Einrichtung erforderlich machen. Insbesondere muss hier der frühzeitigen physio- und ergotherapeutischen Übungsbehandlung ihre herausragende Bedeutung für den Erhalt und die Wiedererlangung der Funktion der Hand beigemessen werden. Gerade im Vergleich zum gestielten Leistenlappen ist in dieser Hinsicht einer freien Lappenplastik aufgrund der besseren postoperativen Mobilisierungsmöglichkeiten der Vorzug zu geben. Die freie Gewebetransplantation hat somit nicht nur aufgrund ihrer sicheren Durchführung und einer mit ca. 95\% hohen Einheilungsrate ihren festen Platz in der wiederherstellenden Handchirurgie erlangt, sondern bietet im Vergleich zu vielen lokalen oder gestielten Lappenplastiken Vorteile in Bezug auf eine frühzeitige Mobilisation und eine auf den Patienten individuell angepasste Defektdeckung. Bei korrekter Indikationsstellung ist die freie mikrovaskuläre Gewebetransplantation heutzutage eine sehr gute Option auch in der primären Rekonstruktion ausgedehnter Weichteildefekte nach Unfällen oder nach Tumorentfernungen im Bereich der Hand.

\section{Literatur}

${ }^{1}$ Levin LS. The reconstructive ladder. An orthoplastic approach. Orthop Clin North Am 1993; 24: 393-409

2 Engelhardt T, Giunta R. Defektdeckung an der Hand. In: Sauerbier M, Eisenschenk A, Krimmer H, Partecke B-D, Schaller H-E, Hrsg. Die Handchirurgie. 1. Aufl. München: Elsevier GmbH; 2015: 452-497

${ }^{3}$ Gottlieb LJ, Krieger LM. From the reconstructive ladder to the reconstructive elevator Plast Reconstr Surg 1994; 93: 1503-1504

${ }^{4}$ Neumeister M, Hegge T, Amalfi A et al. The reconstruction of the mutilated hand. Semin Plast Surg 2010; 24: 77-102

${ }^{5}$ Godina M. Early microsurgical reconstruction of complex trauma of the extremities. Plast Reconstr Surg 1986; 78: 285-292

${ }^{6}$ Lehnhardt M, Hirche C, Daigeler A et al. Weichgewebssarkome der oberen Extremität. Analyse prognoserelevanter Faktoren bei $160 \mathrm{~Pa}-$ tienten. Chirurg 2012; 83: 143-152

7 Müller M, Bickert B, Germann G et al. Weichgewebesarkome an Unterarm und Hand. Plastisch-chirurgische Behandlung. Chirurg 2008; 79: 682-688

8 Taeger CD, Horch RE, Dragu A et al. Perforatorlappen. Eine neue Ära in der rekonstruktiven Chirurgie. Chirurg 2012; 83: 163-171 
${ }^{9}$ Krimmer $H$, Hahn $P$, Lanz $U$. Free gracilis muscle transplantation for hand reconstruction. Clin Orthop Relat Res 1995; 314: 13-18

10 Sauerbier M, Erdmann D, Bickert B et al. Die Defektdeckung an Hand und Unterarm mit dem freien Skapula-Paraskapularlappen. Handchir Mikrochir Plast Chir 2001; 33: 20 25

11 Sauerbier M, Germann G, Giessler GA et al. The free lateral arm flap-a reliable option for reconstruction of the forearm and hand. Hand (New York, NY) 2012; 7: 163-171

12 Flügel A, Kehrer A, Heitmann C et al. Coverage of soft-tissue defects of the hand with free fascial flaps. Microsurgery 2005; 25: 47-53
${ }^{13}$ Megerle K, Sauerbier M, Germann G. The evolution of the pedicled radial forearm flap. Hand (New York, NY) 2010; 5: 37-42

14 Strauch B, Yu H-L. Atlas of Microvascular Surgery. 2nd ed. New York: Thieme; 2006

15 Lister GD, McGregor IA, Jackson IT. The groin flap in hand injuries. Injury 1973; 4: 229-239

16 Adani R, Tarallo L, Marcoccio I et al. Hand reconstruction using the thin anterolateral thigh flap. Plastic Reconstr Surg 2005; 116: 467-473

\section{Marc-Daniel Kunze}

Assistenzarzt

Priv.-Doz. Dr. med.

Isabella M. Mehling

Oberärztin

Prof. Dr. med. Dr. med. habil.

Michael Sauerbier

Chefarzt

Abteilung für Plastische, Hand- und Rekonstruktive Chirurgie

Berufsgenossenschaftliche Unfallklinik Frankfurt am Main

Friedberger Landstraße 430

60389 Frankfurt am Main

marc-daniel.kunze@bgu-frankfurt.de 